\title{
Neurotoxic lesions of the thalamic reuniens or mediodorsal nucleus in rats affect non-mnemonic aspects of watermaze learning
}

\author{
Margriet J. Dolleman-van der Weel · \\ Richard G. M. Morris · Menno P. Witter
}

Received: 18 July 2008/Accepted: 15 December 2008/Published online: 9 January 2009

(c) The Author(s) 2008. This article is published with open access at Springerlink.com

\begin{abstract}
Rats with bilateral neurotoxic reuniens (RE), mediodorsal (MD), hippocampal (HIPP) or sham (SH) lesions were tested in a standard watermaze task, together with unoperated rats. RE-rats and $\mathrm{SH}$-controls readily learned to swim directly to a hidden platform. In contrast, MD-rats displayed a transient deficit characterized initially by thigmotaxis. Like in previous studies, HIPP-rats had long latencies throughout training and displayed more random swims than the other groups. In a memory probe test with the platform removed, SH- and RE-rats approached the correct location relatively directly but, whereas SH-controls persistently searched in the training quadrant, RE-rats switched to searching all over the pool. The MDgroup swam in loops to the platform, but then displayed persistent searching in the training quadrant. The HIPPgroup performed at chance. These distinct patterns indicate that, although their search strategies were different, REand MD-rats had acquired sufficient knowledge about the platform location and could recall information in the probe test. All groups performed well in a subsequent cue test with a visible platform, with RE-rats initially escaping
\end{abstract}

M. J. Dolleman-van der Weel · M. P. Witter

Institute for Clinical and Experimental Neurosciences,

Department of Anatomy and Neurosciences,

VU University Medical Centre, Amsterdam, The Netherlands

M. P. Witter ( $₫)$

Kavli Institute for Systems Neuroscience and Centre for the Biology of Memory, MTFS, Norwegian University of Science and Technology (NTNU), 7489 Trondheim, Norway

e-mail: menno.witter@ntnu.no

R. G. M. Morris

Centre for Cognitive and Neural Systems,

University of Edinburgh, Edinburgh EH8 9JZ, UK faster than the SH- and HIPP-groups, and MD-rats improving from an initially poorer level of performance to control level. This indicates that there were no sensorimotor or motivational deficits associated with any of the lesions. In conclusion, while the RE and MD nuclei seem not to be critical for the learning and memory of a standard watermaze task, they may contribute to non-mnemonic strategy shifting when animals are challenged in ways that do not occur during training.

Keywords Thalamus · Hippocampus · Prefrontal cortex · Behavioural flexibility

\section{Introduction}

Diencephalic (thalamic) amnesia is characterized by deficits resembling those of medial temporal lobe (hippocampal) amnesia or prefrontal dysfunctions (for reviews, Rousseau 1994; Van der Werf et al. 2000, 2003). Thalamic midline nuclei are connected with either the medial temporal lobe, or the prefrontal cortex (PFC), or both. Therefore, thalamic amnesia may result from either (1) disconnecting the temporal and prefrontal systems at the thalamic level, or (2) the loss of specific thalamic contributions to these systems.

Recently, Cain et al. (2006) reported that the medial thalamus of the rat is essential for acquiring watermaze behavioural strategies. However, the role of individual thalamic nuclei is not yet clear. The aim of the present study was to investigate and compare the impact of reuniens (RE) and mediodorsal (MD) lesions upon spatial learning and memory, with their impact on the flexible use of task-relevant strategies.

In the rat, both RE and MD are heavily, and reciprocally connected with the medial (m) PFC (Krettek and Price 
1977; Groenewegen 1988; Vertes 2002, 2004; McKenna and Vertes 2004; Rotaru et al. 2005; Vertes et al. 2006). RE is also heavily connected with hippocampal structures (Herkenham 1978; Wouterlood et al. 1990; Dolleman-Van der Weel and Witter 1996, 2000). It has been proposed that $\mathrm{RE}$ is an important link between mPFC and the hippocampus (Vertes 2006; Vertes et al. 2007), and may play a role in a large-scale limbic network engaged in mnemonic processes (Braak and Braak 1991; Vann et al. 2000). Flämig and Klingberg (1978) conducted, to our knowledge, the only previous behavioural study of RE-lesioned rats. Surprisingly, they reported that learning and memory of a conditioned avoidance task in a Y-maze was unaffected by destruction of RE.

A specific role for MD in cognitive processes is still controversial (e.g., Markowitsch 1982; Stokes and Best 1990; Peinado-Manzano and Pozo-Garcia 1996; Chauveau et al. 2005; Mitchell et al. 2007a, b). Lesions of MD can result in deficits that resemble those of mPFC lesions (Hunt and Aggleton 1991; McAlonan et al. 1993). Hunt and Aggleton (1998) suggest that acquisition deficits arising from MD lesions may be due to disruption of processes that interact with task performance (e.g., strategy learning, response flexibility), rather than with mnemonic processes.

In the present study, we examined whether RE and/or MD input is essential for the functioning of the hippocampal/mPFC memory systems, or for the normal behavioural expression of information acquired by these memory systems. Based on changes in c-fos activity, Vann et al. (2000) suggested that RE plays a role in working memory. However, c-fos imaging yields complex and sometimes controversial results (e.g., Aggleton et al. 2000; Bertaina-Anglade et al. 2000; Jenkins et al. 2003; Santin et al. 2003), while the precise role of c-fos in memory formation and the underlying mechanisms remain unknown (Herrera and Robertson 1996; Zhang et al. 2002). Therefore, we used a conventional (reference memory) watermaze task, known to be sensitive to dysfunction of both the hippocampal and prefrontal systems, with each system mediating different aspects of watermaze learning. Whereas the hippocampal formation is engaged in the spatial aspects of learning and memory, the role of mPFC in this task appears to involve behavioural flexibility and the execution of spatial strategies, rather than encoding or storage of spatial information (De Bruin et al. 1994; Ragozzino et al. 1999a, b; De Bruin et al. 2001; Lacroix et al. 2002; Passetti et al. 2002; Ragozzino et al. 2003). Therefore, a RE lesion, that could affect both the hippocampal and mPFC memory systems, might be expected to cause a mixture of hippocampal (spatial learning/memory) and mPFC (behavioural flexibility/strategy learning) related impairments. An MD lesion, likely affecting primarily mPFC memory functions more specifically, was expected to result in an acquisition deficit in behavioural flexibility when task conditions change.

\section{Materials and methods}

\section{Subjects}

We used 42 male Lister hooded rats (weighing 280-400 g at the time of surgery) from breeding stock in the University of Edinburgh. They were housed individually in plastic cages with ad libitum access to food and tap water at all times. A normal $12 \mathrm{~h}$ dark/light cycle was maintained, with all behavioural training and testing carried out in the light phase.

All experiments described here have been conducted in accordance with the European Communities Council Directive (1986), and with the approval of the local Animal Experimentation Committee of the VU University medical centre, Amsterdam. All efforts were made to minimize any suffering and the number of animals used.

\section{Surgery}

Restricted lesions in RE and MD were created by injecting small amounts of ibotenic acid (IBO, Cambridge Research Biochemicals, Cambridge, UK) into the respective nuclei, resulting in local cell death with minimal damage to fibres of passage (Köhler and Schwarcz 1983). The animals were anaesthetized with tribromethanol $(10 \mathrm{ml} / \mathrm{kg}$, i.p.) and placed in a Kopf stereotaxic frame. The skull was exposed and burr holes were made to accommodate injections of IBO $(10 \mathrm{mg} / \mathrm{ml}$ phosphate buffered saline), applied with the use of a glass micropipette that was glued to the end of the needle of a $1 \mu$ l Hamilton syringe. IBO was infused slowly in a volume of $0.10 \mu \mathrm{l}$ per injection site over a period of $10 \mathrm{~min}$. After leaving the pipette in situ for another $5 \mathrm{~min}$, to ensure diffusion of IBO into the target structure, it was slowly retracted. Stereotaxic coordinates were derived from Paxinos and Watson (1986), aligned with respect to bregma $(\mathrm{Br})$, the midline of the superior sagittal sinus (medial-to-lateral, ML), and the surface of the dura (dorsal-to-ventral, DV). Because RE is a very small nucleus, difficult to lesion selectively, the RE-group contained more animals than the other groups. Rats in the REgroup ( $n=12$ ) received bilateral injections in the rostral as well as in the caudal part of the nucleus (rostral $\mathrm{RE}, \mathrm{Br}$, $-1.80 \mathrm{~mm}$; ML, $2.0 \mathrm{~mm}$, at an angle of $15^{\circ}$ in the coronal plane; DV, $6.9 \mathrm{~mm}$; caudal $\mathrm{RE}, \mathrm{Br},-2.30 \mathrm{~mm}$; ML, $1.4 \mathrm{~mm}$, at an angle of $10^{\circ}$ in the coronal plane; DV, $7.0 \mathrm{~mm}$ ). Rats in the MD-group $(n=6)$ received bilateral injections that were placed according to the RE coordinates, except that at the rostral injection sites the pipette 
was lowered to a depth of $4.8 \mathrm{~mm}$, and at the caudal sites to $5.2 \mathrm{~mm}$. Sham (SH)-controls underwent anaesthesia/surgical procedures that were similar to those for RE- and MD-rats, except that (1) in the SH-hippocampus-group (SH-HIPP, $n=6$ ) a needle was lowered through the dorsal hippocampus, a structure implicated in spatial memory (Moser et al. 1993; Moser and Moser 1998), but no IBO was injected (i.e., a procedure, which causes mechanical hippocampal damage that is identical to that necessarily caused in creating the RE and MD lesions), and (2) in the SH-dura-group $(n=6)$ only the dura was cut.

Under certain training conditions, animals with damage to the hippocampal formation are capable of acquiring a place response (Whishaw and Tomie 1997; Gerlai et al. 2002; Pouzet et al. 2002). Therefore, a HIPP-group was added to establish the degree of hippocampal related spatial impairment under our training and test conditions. Rats in the HIPP-group $(n=6)$ received 26 injections along the entire longitudinal axis of the hippocampus, completely destroying the hippocampal formation (procedure previously described by Jarrard 1989). Following surgery all rats were allowed 2 weeks recovery.

It is well known that anaesthetics can affect cognitive functions (e.g., Culley et al. 2003, 2004; Baxter et al. 2008). There are no specific reports whether or not tribromethanol affects learning and memory, but it can have various side effects (e.g., Zeller et al. 1998; Thompson et al. 2002; Meyer and Fisch 2005; Lieggi et al. 2005) which may affect behaviour. In order to control for any effect of the anaesthesia/surgical procedure, unoperated rats (UNOP-group, $n=6$ ) were added as well.

\section{Behavioural training and testing procedures}

All rats were well handled before being trained in a $2 \mathrm{~m}$ open-field watermaze, filled with water $\left(25 \pm 1^{\circ} \mathrm{C}\right)$ made opaque by the addition of powdered milk. An escape platform (10 $\mathrm{cm}$ in diameter) was placed at a fixed position in one of the quadrants of the pool, arbitrarily designated NE, NW, SE and SW. The pool was situated in a diffusely illuminated room, containing prominent extra-maze cues to enable the rats to learn the platform's location. A curtain hanging from the ceiling could be drawn around the pool to obscure the room cues. A video camera mounted on the ceiling was connected with a computerized tracking system (HVS image analyser and Acorn Archimedes computer; Hawk Track, Watermaze program) to monitor and store the swim paths of the animals for off-line analysis. In general, at the start of each trial the rat was placed in the pool facing the wall, and then allowed to search for the submerged (i.e., $1 \mathrm{~cm}$ below the water surface) escape platform for a maximum of $120 \mathrm{~s}$. The rat remained on the platform for $30 \mathrm{~s}$, after which the next trial was run immediately. If the animal failed to escape from the water within $120 \mathrm{~s}$, it was guided to the platform by hand. After finishing the trials, the animals were dried and warmed before being returned to their home cage.

Pre-training (day 1) consisted of 4 swim trials to familiarize the animals with the general procedures of the task (e.g., searching the pool, climbing onto the platform). Spatial learning was prevented by drawing curtains around the pool to exclude room cues. The starting point and position of the submerged platform differed per trial.

Spatial training (days 2-4) consisted of 18 trials (i.e., 6 trials/day), with room cues visible. For each group, half of the rats were trained to find the submerged platform at a fixed location in the NE quadrant, for the other half of the group the platform was located in the SW quadrant. The starting points $(\mathrm{N}, \mathrm{S}, \mathrm{E}$ or $\mathrm{W})$ were varied in a semi-random way across trials.

A single transfer (or "memory probe") test (day 5) was run during which the escape platform was removed from the pool, and the rats allowed a free swim of $60 \mathrm{~s}$. Performance in the probe test is generally accepted to reflect the rats' memory for the learned platform location, which is behaviourally shown as the proportion of time spent in the training quadrant.

Finally, a cue test consisting of four trials (day 6) was given with the platform visible (i.e., $1 \mathrm{~cm}$ above the water surface), and curtains surrounding the pool to exclude extra maze cues. This test served a dual purpose: (1) due to a change in task demands, performance in the cue test will reflect the animals' ability to switch to a different problem solving strategy, and (2) the cue test is assumed to reflect the occurrence of any gross sensorimotor and/or motivational deficiencies.

\section{Data collection and statistics}

The behaviour of the animals was analyzed off-line, focusing upon performance during the spatial training (submerged platform), the transfer test (platform removed), and the cue test (visible platform). Parameters computed by the software were: escape latency, path length, swim speed, quadrant time, and directionality. We also performed an analysis of the swim paths recorded during the spatial training phase. This was done in order to examine whether the different groups made use of particular search strategies. Distinctive swim paths were categorized according to a system that was modified and expanded after Whishaw and Jarrard (1995). We distinguished the following categories: (A) edge, (B) random, (C) circle, (D) loop, (E) direct, $(F)$ indirect, and $(G)$ near miss (for typical examples, see Fig. 4a). Accordingly, the 18 swim paths of each rat were blindly analyzed by an observer and attributed to these categories. Whenever a path showed multiple 
characteristics, it was attributed to the category that dominated the swim. For each group the number of swim paths per category per group across spatial training trials was used for statistical analyses. For representation in a figure, the scores were normalized by expressing them as percentage of swims belonging to a particular category.

Statistical analyses used an ANOVA for overall comparison, and Dunnett's test for comparison between groups. Statistical packages used were ALICE (System for manipulating and analyzing multidimensional data) and SPSS (Statistical Package for the Social Sciences). Significance was set at $P<0.05$.

Variability in performance within groups may be related to differences in lesion size (including inadvertent damage to adjacent structures). This possibility was investigated using the Spearman rank correlation coefficient. During the training trials the most efficient strategies are the relatively direct $(E+F+G)$ routes. These strategies are associated with short escape latencies, and clearly reflect the rats' knowledge of the hidden platform location. Therefore, the ranking of smallest to largest lesion was compared to the ranking of most to least frequent use of $E+F+G$ paths (i.e., best to worst performance, respectively). In addition, the ranking according to lesion size was compared to ranking of the highest to lowest training quadrant time in the transfer test (i.e., a memory measure).

\section{Histology}

At the end of the experiment, the animals received an injection of sodium pentobarbital (Euthatal, $200 \mathrm{mg} / \mathrm{kg}$, i.p.) and were transcardially perfused with physiological saline and $10 \%$ formalin fixative. The brains were removed and stored in fixative for at least $24 \mathrm{~h}$. Subsequently, they were cryoprotected in $2 \%$ dimethyl sulfoxide and $20 \%$ glycerin in phosphate buffer. On a freezing microtome the brain tissue was cut to either coronal (RE-, MD-, SH- and UNOP-groups) or horizontal (HIPP-group) sections of $40 \mu \mathrm{m}$ thickness. Every fifth section was Nissl-stained with cresyl violet or thionin, and analyzed to determine the extent of the IBO lesions in the RE-, MD-, and HIPP-rats, and the mechanical damage caused by the sham-lesioning procedure in the SH-HIPP and SH-dura controls.

\section{Results}

Histological observations

The brains of the RE-rats $(n=12)$ showed that RE was completely destroyed in all but one animal. In the latter case, approximately $90 \%$ of the nucleus was lesioned, leaving only its very rostral part in tact. In two rats the lesion was strictly confined to RE. In the other ten animals, due to leakage of IBO along the injection tract, the extent of the lesion ranged from a minor involvement of the rhomboid, anteromedial, interanteromedial and gelatinosus nuclei $(n=4)$, to a moderate damage in the midline including a part of the intralaminar central medial nucleus $(n=6)$. In most cases, some mechanical damage was noticed in MD. Commonly, the tract through the overlying hippocampus was accompanied by a restricted cell death and gliosis in CA1 and/or the dentate gyrus (not illustrated). Because the variability of RE lesions was relatively small, and later statistical analysis did not show any correlation between lesion size and behaviour (see below), all animals were included in the RE-group. The extent of the smallest and largest RE-lesion is schematically represented in Fig. 1a. A photomicrograph of a lesion confined to RE, taken approximately at the level illustrated in Fig. 1a (bregma-2.30), is shown in Fig. 2a, $\mathrm{a}^{\prime}$.

In all MD-rats $(n=6)$ the lesion was centred in MD. The estimated extent of the MD-lesions, however, varied from 50 to $80 \%$ of the nucleus. In all cases, the most caudal part of MD appeared to be intact. In threes rats, the intralaminar and paratenial/paraventricular nuclei showed partial cell loss as well. In all animals but the one with the smallest MD-lesion, we noticed some damage in the anterodorsal nucleus. Inadvertent hippocampal damage along the injection tract (not illustrated) was comparable to that noticed in the RE-group. The extent of the smallest and largest MD-lesion is schematically represented in the Fig. 1b. A photomicrograph of the largest MD lesion, taken approximately at the level illustrated in Fig. 1b (bregma $-2.30)$, is shown in Fig. $2 b, b^{\prime}$.

The brains of all HIPP-rats $(n=6)$ displayed a massive destruction of the dentate gyrus, the CA fields and the subiculum, although in three cases partial (restricted) sparing of neurons in the intermediate hippocampal region was noticed. In addition, the entorhinal cortex showed minor damage in all but one animal, whereas the neocortex (i.e., mainly visual areas overlying the hippocampus) was moderately, but comparably damaged in all cases (not illustrated). In all six rats, the thalamus was unaffected. Figure 1c shows the extent of the smallest and largest HIPP-lesion. Photomicrographs in Fig. 2c illustrate the smallest (i.e., least complete) HIPP lesion at a dorsal (left) and ventral (right) level, approximately at interaural 6.90 and 3.90, respectively (see Fig. 1c).

The brains of the rats in the SH-dura- $(n=6)$ and SHHIPP-groups $(n=6)$, showed minimal cell loss and/or some gliosis in the superficial cortical layers. In the SHHIPP-group we also noticed restricted damage in CA1 and the dentate gyrus, directly bordering the needle tract (not illustrated). 
Fig. 1 Schematical representation of smallest (light grey area) and largest lesions (dark grey area) of RE (a), MD (b), and HIPP (c) in a series of sections at four rostro-to-caudal (a, b), and dorsal-to-ventral levels (c) through the rat brain. Abbreviations CA1-3 cornu ammonis field 1-3, $C L$ centrolateral nucleus, $C M$ centromedial nucleus, $D G$ dentate gyrus, $E C$ entorhinal cortex, $f$ fornix, $H I P P$ hippocampal formation, $M D$ mediodorsal nucleus, $P C$ paracentral nucleus, $P t$ paratenial nucleus, $R E$ nucleus reuniens, $S u b$ subiculum

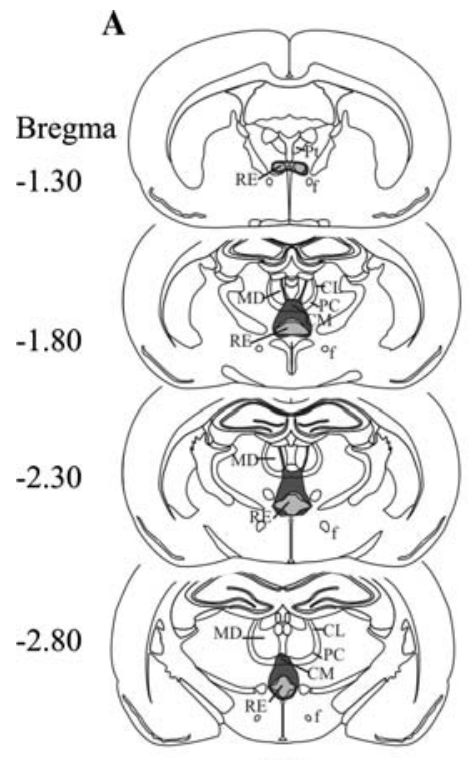

RE
B

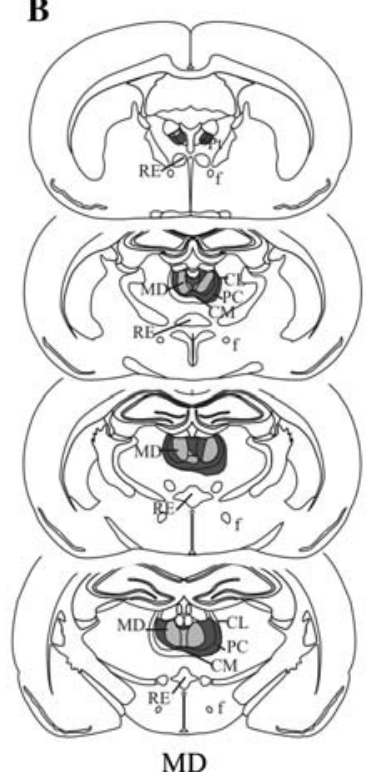

C

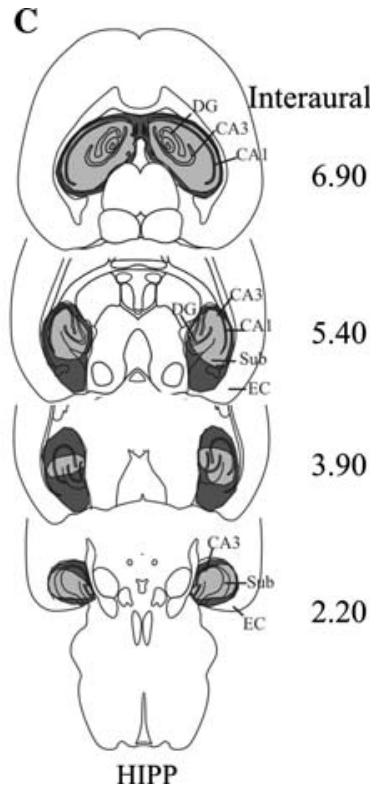

Based on these histological observations, none of the lesioned animals had to be excluded.

Behavioural observations

During training and testing, it became clear that one of the $\mathrm{SH}$-dura rats displayed a serious visual deficit. Therefore, this rat was excluded from the behavioural analyses, reducing the SH-dura-group to $n=5$. All the remaining rats swam in a normal way, using the adult swimming posture. They had no difficulty climbing onto the platform, or using it as a suitable means of escape from the water.

\section{Spatial training}

Because of a lack of significant differences between the SH-HIPP- and SH-dura-groups (data not shown), they were combined into a single sham lesion (SH) group $(n=11)$. Thus, all following analyses dealt with five groups, i.e., UNOP-rats, and RE-, MD-, HIPP-, and SH-lesioned animals.

The 18 training trials (days 2-4) were grouped into six blocks of three trials each (i.e., 2 blocks/day) and analyzed for escape latencies. Figure 3 shows the mean escape latencies of the five groups across blocks. An ANOVA revealed that these latencies differed significantly between groups $[F(4,36)=5.87, P<0.001]$, blocks $[F(5,180)=$ 44.38, $P<0.001]$ and there was a significant groups $\mathrm{x}$ blocks interaction $[F(20,180)=2.17, P<0.005]$, the latter likely due to the long latencies of the MD- and HIPPgroups. Subsequently, we compared group latencies per block, showing that on day 1 (block 2) the SH-group had longer escape latencies than the UNOP-group $(P<0.05)$.
This small, yet significant difference (as well as a significant difference in transfer test performance, see below) indicated an effect of the anaesthesia/surgical procedure that should be taken into account when analyzing the performance of the three lesion groups. Therefore, the SHand UNOP-rats were not combined into one group. In all analyses, the behaviour of the RE-, MD-, and HIPPlesioned rats was compared to that of the SH-controls, as well as to each other; comparisons between lesion groups and UNOP-rats were not conducted.

Further comparison of group latencies per block revealed that, compared to $\mathrm{SH}$-controls, the MD-group displayed longer latencies on the first block of each training day (block 1: $P<0.005$; block 3: $P<0.05$; block 5: $P<0.025)$, whereas the RE-group was not significantly different from the SH-group (block 3: $0.10>P>0.05$, trend). The HIPP-group had long latencies throughout spatial training (HIPP vs. SH; blocks 2 and 3, $0.10>P>0.05$, trend; block 4, $P<0.001$; block 5 , $P<0.05$; block 6, $P<0.005)$. A comparison of the three lesion groups revealed that the RE-group was significantly faster than the MD- and HIPP-groups (RE vs. MD: blocks 1 and $4, P<0.005, P<0.05$, respectively; RE vs. HIPP: blocks 4, 5, and 6, $P<0.05, P<0.05$, and $P<0.0025$, respectively).

There was no overall difference in swim speed [mean speed $(\mathrm{m} / \mathrm{s}) \pm$ SEM: RE, $0.29 \pm 0.003 ; \mathrm{MD}, 0.29 \pm 0.01$; HIPP, $0.30 \pm 0.01 ; \quad \mathrm{SH}, 0.28 \pm 0.01 ;$ UNOP, $0.29 \pm$ $0.01\}$, and the analysis of path length therefore closely followed the pattern of the analysis of escape latencies (data not shown).

The different lesions had a distinct effect on the use of distinctive swim strategies. In Fig. 4a, the categories A-G 
Fig. 2 Photomicrographs of representative examples of Nissl-stained coronal sections illustrating a RE-lesion (a), a MD-lesion (b), and horizontal sections of a HIPP-lesion (c) at a dorsal (left) and a ventral level (right). Boxed areas in $\mathbf{a}$ and $\mathbf{b}$ are shown at higher magnification in $\left(\mathbf{a}^{\prime}\right)$ and $\left(\mathbf{b}^{\prime}\right)$, respectively. The lines delineate the lesioned area. In case of HIPP-lesions (c), almost all of the structure has disappeared, similar to what is shown for the left side of the (bilateral) RElesion $\left(\mathbf{a}, \mathbf{a}^{\prime}\right)$. On the right side of the RE-lesion, as well as within the (bilaterally) lesioned MD (b, $\left.\mathbf{b}^{\prime}\right)$ there are numerous Nissl-stained astrocytes present, yet only a few if any surviving neurons can be detected

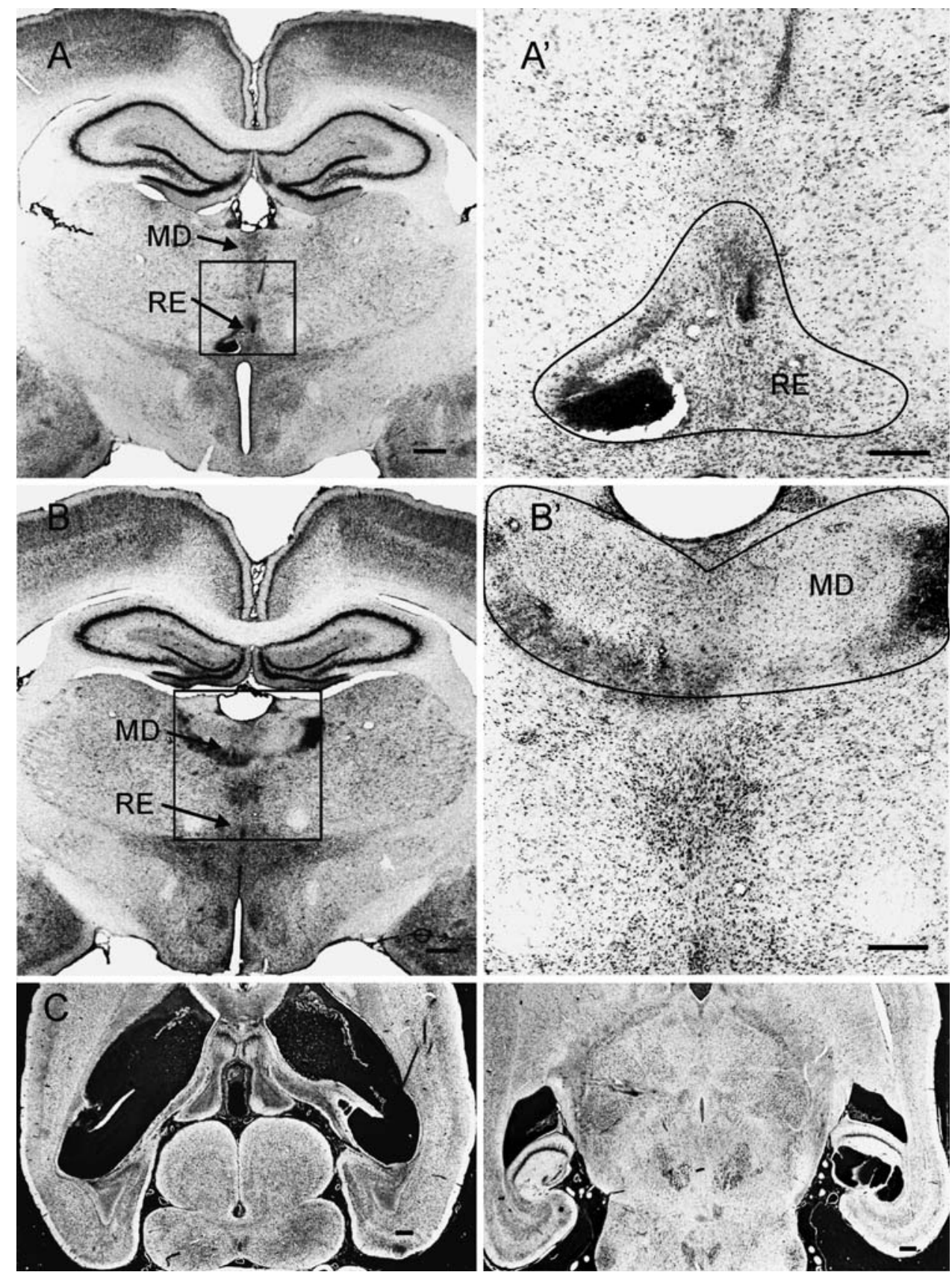

(see "Materials and methods") are illustrated by examples of swim paths that show the range for each category. The differential use of these search strategies across the 3 days of spatial training is represented in Fig. 4b. The UNOP-, SHcontrol, and RE-rats quickly learned to swim a direct path (E) to the hidden escape platform. In contrast, particularly on the first block of trials of each day, the MD-group persistently used the least efficient strategy [i.e., edge swimming (A), associated with long latencies, see also Fig. 3], with little chance to encounter the submerged platform. Their behaviour gradually changed to swimming loops (D) and indirect routes $(\mathrm{F})$. The HIPP-group displayed the highest percentage of random paths (B) throughout training, although these were often alternated with circle swims (C) and loops (D). An ANOVA revealed that group differences in the overall use of strategies reached significance for the categories edge (A) $[F(4,36)=6.89, P<0.001]$, random $(\mathrm{B})[F(4,36)=9.39$, $P<0.001]$, loop (D) $[F(4,36)=2.65, P<0.05]$, and direct (E) $[F(4,36)=7.14, P<0.001]$. Further analyses showed that both SH-controls and RE-rats swam significantly more direct routes (E) than the MD- and HIPP-groups (SH vs. MD, $P<0.005$, and SH vs. HIPP, $P<0.001$; RE vs. MD, $P<0.05$, and RE vs. HIPP, $P<0.0025$, respectively). In turn, the MD-group displayed significantly more edge (A) 
Spatial training

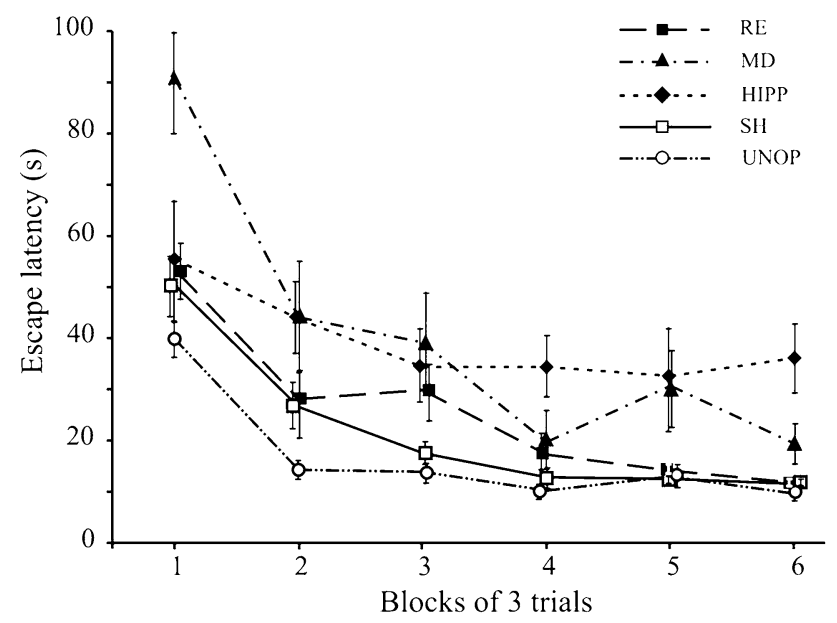

Fig. 3 Mean escape latencies against blocks of three trials each during spatial training, days 2-4. The SH-control group had significantly longer latencies than the UNOP-group on block 2 . Notice the long latencies of the MD-group during the first block of each day (i.e., blocks 1, 3, and 5), and their improvement on the second block of each day. The HIPP-group displayed long latencies throughout training, whereas the RE-group was not significantly different from the SH-controls

swims than the SH-, RE- and HIPP-rats (MD vs. SH, $P<0.005 ;$ MD vs. RE, $P<0.001 ;$ MD vs. HIPP, $P<0.025)$, whereas the HIPP-group swam more random paths (B) than the SH-, RE-, and MD-animals (HIPP vs. SH, $P<0.001$; HIPP vs. RE, $P<0.001$; HIPP vs. MD, $P<0.005$ ). Regarding the category loop (D), differences were only found between the three lesion groups, i.e., the RE-group swam less loops than the MD- and HIPP-groups (RE vs. MD, $P<0.01$; RE vs. HIPP, $P<0.005$ ).

\section{Transfer test}

To examine the rats' memory for the learned platform location, a single trial was run during which the platform was removed from the pool. An overall ANOVA showed a highly significant difference across groups in the distribution of time spent in the four quadrants $[F(3,108)=34.08$, $P<0.001]$ and a group $\mathrm{x}$ quadrant interaction $[F(12,108)=3.35, P<0.001]$. A separate analysis was conducted of time spent in the training quadrant only, showing a highly significant difference between groups $[F(4,36)=7.45, P<0.001]$. Further analyses revealed that the SH-controls spent significantly less time in the training quadrant than the UNOP-group $(P<0.05)$. The HIPP-group was significantly impaired ( $\mathrm{SH}$ vs. HIPP, $P<0.05$ ), with a training quadrant time indistinguishable from chance level (see Fig. 5, quadrant time expressed in percentages: $26.7 \% \pm 3.9$; chance $=25 \%$ ). Comparison of the SH- versus the RE- and MD-groups did not reach significance, although the RE-group (see Fig. 5, training quadrant time of $33.2 \% \pm 1.7$ ) performed rather poorly (SH vs. RE, $0.10>P>0.05$, trend). Comparison of the three lesion groups, however, revealed that the RE-rats spent more time in the training quadrant than the HIPPanimals $(P<0.05)$.

There was an overall difference in path length $[F(4,36)=5.42, P<0.0025]$, with significantly longer paths for the lesioned rats (i.e., versus SH-controls: HIPP, $P<0.05$; RE, $P<0.001$; MD, $P<0.05$ ). The SH-controls did not differ on this measure from the UNOP-rats (n.s.). We also found an overall difference in swim speed $[F(4,36)=4.24, P<0.01)]$, with the three lesion groups swimming slightly faster (mean speed $0.33 \pm 0.02 \mathrm{~m} / \mathrm{s}$ ) than the SH- and UNOP-rats (mean speed $0.29 \pm 0.01$, and $0.28 \pm 0.01 \mathrm{~m} / \mathrm{s}$, respectively). This was due to the animals of control groups dwelling in the vicinity of the platform location, frequently stopping and turning around, rather than differences in the actual speed of swimming when it occurred. Figure 5 also shows, for each group, a representative swim path during the transfer test, with the initial approach of the platform location represented by the thickened line. UNOP- and SH-rats swam relatively direct (i.e., using $\mathrm{E}+\mathrm{F}+\mathrm{G}$ paths) to the learned platform location, yet whereas the UNOP-group persisted in searching in the training quadrant, the SH-group gave up after some time and searched in a larger area of the pool. All RE-rats also swam relatively direct to the correct location. However, when the platform was not encountered, unlike SH-controls, they carried on swimming to search all over the pool. In contrast, MD-rats swam mainly in loops to the platform and then, once they got there, continued searching in the training quadrant. HIPP-rats swam mainly in circular paths, often crossing the former platform location and possibly using the wall as reference for their search.

We also included an assessment of whether the animals were heading for the platform $50 \mathrm{~cm}$ away from their starting point (i.e., a directionality measure). A trend but no significant difference was observed $[F(4,36)=2.23$, $0.10>P>0.05$; ranking from best to worst: UNOP, $\mathrm{SH}$, RE, MD, HIPP], reflecting that the UNOP- and SH-rats showed a tendency to be heading more accurately for the platform location than the lesion groups. Unfortunately, variability in this measure makes it difficult to secure clear cut results for "heading-direction".

\section{Cue test}

A final test with a visible platform was conducted on day 6 (see Fig. 6). An ANOVA showed a significant difference in overall latencies for groups $[F(4,159)=3.70, P<0.01]$, trials $[F(3,108)=6.56, P<0.001]$, and a groups $\times$ trials interaction $[F(12,108)=1.96, P<0.05]$. No differences 
Fig. 4 Search strategies during spatial training. a Examples of swim paths illustrating the range for each category. b Differential use of strategies across blocks of trials, as indicated by the percentage of swim patterns used by the five groups. The exceptional display of edge swimming by MD-rats closely followed their pattern of escape latencies (see Fig. 2). HIPP-rats displayed the most random swims throughout training, whereas SH-, RE- and UNOPrats rapidly learned to swim mainly direct paths, occasionally alternated with near miss and indirect paths
A

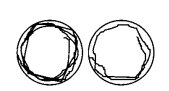

Edge (A)

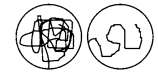

Random (B) Categories of swim paths
B
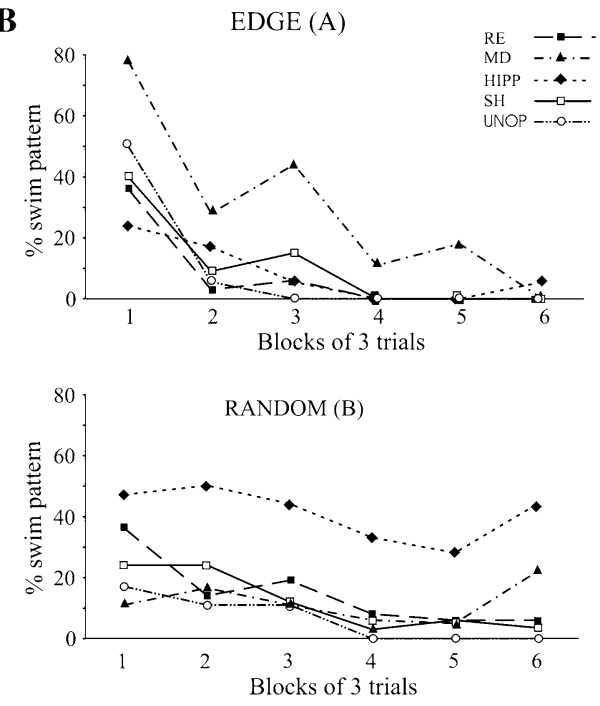

CIRCLE (C)
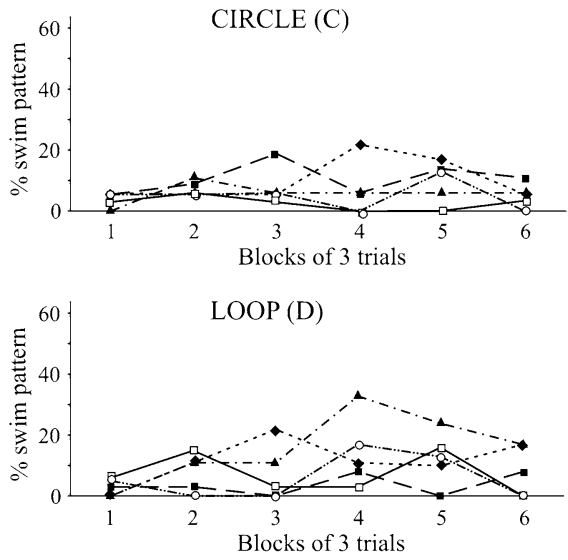

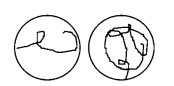

Loop (D)

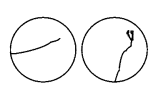

Direct (E)

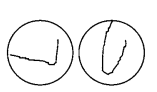

Indirect $(\mathrm{F})$

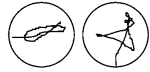

Near miss $(\mathrm{G})$
$\operatorname{DIRECT}(\mathrm{E})$
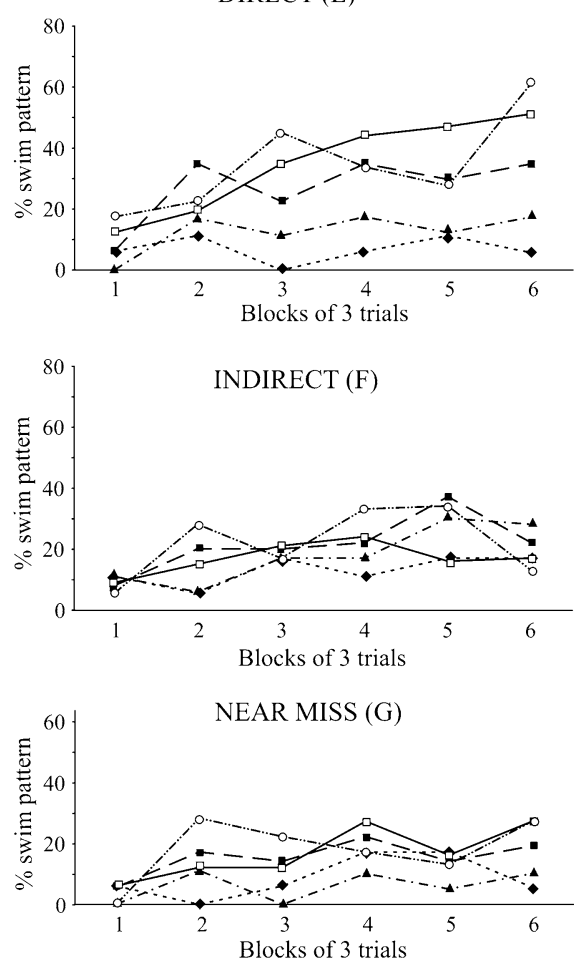

were found between SH-controls, UNOP-, and HIPPgroups. However, RE-rats had significantly shorter latencies than SH- and HIPP-rats on trial 2 (RE vs. SH, $P<0.01$; RE vs. HIPP, $P<0.05)$. The extremely long latencies of the MD-group (trials 1 and 2, likely due to swimming mainly loops and indirect paths, ignoring the visible platform and searching for the hidden one) failed to reach significance versus both the $\mathrm{SH}$-controls and the REgroup $(0.10>P>0.05$, trend), most likely due to the rather large variation. Therefore, cue test analyses were also conducted using non-parametrical tests. This yielded similar statistical results (data not shown).

\section{Relation between lesion size and behaviour}

On the acquisition measure (i.e., use of $\mathrm{E}+\mathrm{F}+\mathrm{G}$ paths, associated with short escape latencies) there was no significant correlation with lesion size for any of the lesion groups ( $\mathrm{RE}, \mathrm{rs}=-0.2 ; \mathrm{MD}, \mathrm{rs}=0.7$; HIPP, $\mathrm{rs}=-0.3$ ), although there was a slight tendency towards a positive correlation in the MD-group. On the memory measure (i.e., training quadrant time in the transfer test) we found also no significant correlation ( $\mathrm{RE}, \mathrm{rs}=-0.4 ; \mathrm{MD}$, rs $=-0.4$; HIPP, rs $=-0.6)$. The lack of significant correlations largely reflects the lesions being relatively complete as intended.

\section{Discussion}

We examined the role of the thalamic RE and MD nuclei in spatial learning and memory, using a conventional (reference memory) watermaze task. The main findings were that (1) RE-lesioned rats, like SH-controls, rapidly learned the 


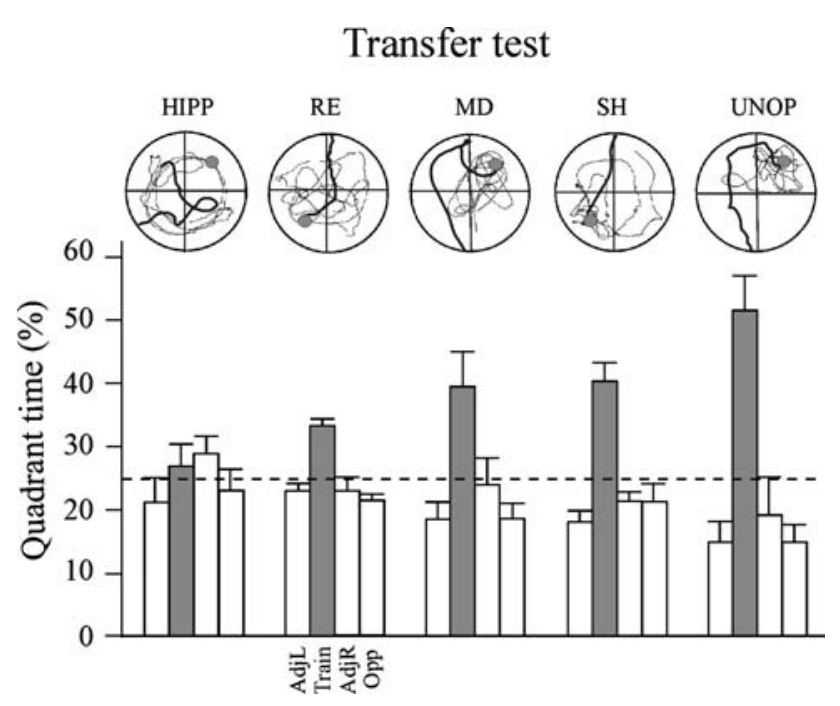

Fig. 5 Distribution of time (expressed as mean percentage) spent in the 4 quadrants of the pool during the transfer test, with emphasis on time spent in the training quadrant (grey bars; the dotted line represents chance level $=25 \%$ ). The statistical analysis of actual quadrant time revealed that the SH-group spent significantly less time in the training quadrant than the UNOP-group, but more time than the HIPP-group. Despite of rather poor performance in the RE-group, RE- and MD-rats were not significantly different from SH-controls. For each group a representative swim path is shown; grey dots mark the location of the (removed) platform. The initial part of the swim path is marked by the thickened line, illustrating the differences in approaches of the learned location between groups. While UNOP-rats persistently searched in the training quadrant, SH-rats also searched at the correct location, but gave up after some time and then swam over a larger area of the pool. RE-rats swam directly towards the learned location, but when the platform was not encountered they switched immediately to searching all over the pool. MD-rats swam in loops towards the platform and then kept searching that area. HIPP-rats mainly circled at a certain distance from the pool wall, often crossing the former platform location. train training quadrant, adj/l adjacent left, $a d j / r$ adjacent right, opp opposite

task and swam mainly direct paths to the invisible platform, whereas MD-lesioned rats displayed a transient acquisition deficit, characterized initially by perseveration of edge-swims; (2) when the platform was removed (probe test), RE-lesioned rats swam relatively direct to the correct location, but did not stop and search locally. Instead, they carried on swimming around the pool. In contrast, MDlesioned rats swam in loops to the former platform location, but then displayed persistent searching in the training quadrant; (3) when task conditions were altered (visible platform test), RE-lesioned rats escaped initially faster than SH-controls. MD-lesioned rats, however, initially displayed abnormalities in the paths taken to the escape target, but eventually recognized and used the visible platform as refuge. These findings argue against a role for RE and MD in mnemonic aspects of spatial learning, but instead point to a role in the behavioural strategy used to express spatial

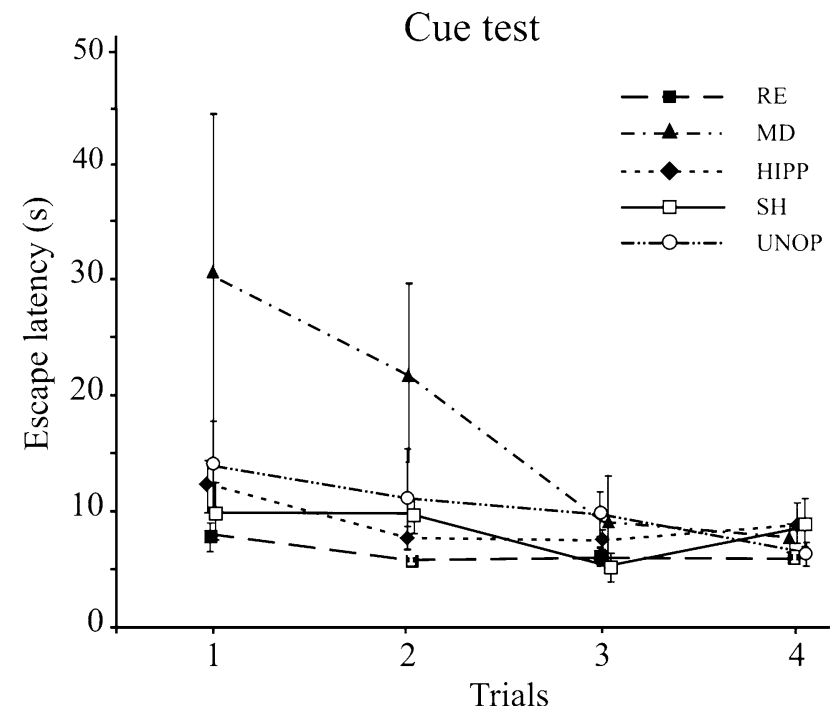

Fig. 6 Mean escape latencies in the cue test (visible platform). The RE-group displayed significantly shorter latencies than the SH- and HIPP-groups on trial 2. The initially poor performance of the MDgroup (trials 1 and 2) did not reach significance versus the SHcontrols, and showed a trend $(0.10>P>0.05)$ versus the RE-group

information and the flexibility with which environmental changes can be accommodated.

\section{Effects of anaesthesia/surgical procedure}

Small, yet significant differences between the SH- and UNOP-groups were found in two measures: (1) SH-rats had longer escape latencies in the early phase of spatial training, and (2) in the transfer test they spent less time in the training quadrant. These differences between the SHand UNOP-groups imply that (at least with our training and test protocols) sustained tribromethanol anaesthesia and/or a sham lesion is sufficient to bring about a partially impaired performance that should be taken into account when examining lesion effects. Therefore, in this study the SH-group (and not the UNOP-, or a combined SH/UNOPgroup) was considered the appropriate control for comparison with the RE-, MD-, and HIPP-groups.

\section{Effects of hippocampal versus thalamic lesions}

In line with previous reports, HIPP-rats were considerably impaired and their escape latencies improved only slightly across training trials. Most conspicuous was that, unlike any of the other groups, HIPP-rats displayed random swims throughout acquisition that, eventually, often alternated with circling. These strategies also characterized the search of the HIPP-group in the transfer test. Despite of a clear navigational deficit, their "platform biased circling" suggested that they had acquired at least some knowledge of 
the (now absent) platform location, but probably used distance to the wall to guide their search rather than integrating spatial cues in a normal way (e.g., Morris et al. 1990; Whishaw and Jarrard 1995; Whishaw et al. 1995; Whishaw and Tomie 1997; Pouzet et al. 2002). These results show that the present behavioural protocol is sensitive to dysfunction of the hippocampal formation.

We therefore expected that depriving the entorhinalhippocampal circuitry from RE input, which had been hypothesized to be crucial for (hippocampal-related) spatial aspects of watermaze learning, should in principle be detected with our training and test procedure. Surprisingly, the RE-group displayed no lasting acquisition deficit. They learned the task rapidly and, like SH-controls, swam with significantly more direct routes to the platform than the HIPP- and MD-groups. This indicates that RE afferents to the hippocampal formation may not be critical for either hippocampal-related spatial learning and memory encoding, or the learning of mPFC-related procedural strategies necessary for a reference memory watermaze task. In contrast, the MD-group displayed a transient acquisition deficit with a specific pattern of long latencies, which closely matched the pattern of gradual decline in edge-swims across blocks of training. Overall, the MD-group showed significantly more swimming at the edge (sometimes referred to as "thigmotaxis") than the SH-, RE- and HIPP-groups. Commonly, thigmotaxis is only noticed during the first trials after introduction (i.e., in this study during pretraining) to the pool. When this behaviour appears ineffective, the animals will subsequently explore the pool by a rapid shift to other strategies (e.g., random, loop) and after only a few spatial training trials they have learned to swim directly to the correct location. Figure 4 clearly illustrates that, in the first block of spatial training, MD-rats displayed far more edge swimming than the four other groups. This suggests that, in this respect, the MD-group had experienced a less beneficial effect from the pretraining trials than the other lesion and control groups. Obviously, perseveration of edge-swims will hamper the rate of learning, because MD-rats will then have less chance to encounter the hidden platform and so be rewarded for a more efficient navigational strategy. It has been reported that mice with large MD lesions extending into $\mathrm{CM}$, showed increased fear reactivity (elevated-plus maze, GO/NOGO temporal alternation tasks, Chauveau et al. 2005). The latter authors suggested that a cognitive deficit of MD-lesioned animals (characterized by a difficulty in maintaining an alternation rule with procedural variance) could stem primarily from increased fear. We cannot entirely rule out the possibility that the acquisition deficit of the MD-group was (partly) due to an increased level of fear. Yet, to our knowledge, there are no reports available to support the idea that MD- lesioned rats can display "fear for the open field", which otherwise might have contributed to the presently observed perseveration of edge-swims. The long latencies of the MD-group during acquisition do not, however, necessarily imply a spatial learning/memory deficit (see below).

Despite of normal acquisition, the RE-group spent an unexpectedly poor percentage of their time in the training quadrant in the transfer test. In the initial phase of the test, all RE-rats (like SH-controls) approached the correct location relatively direct, indicative of normal learning and memory. Unlike SH-controls, when the platform was not encountered at the learned location, RE-rats continued swimming and searched all over the pool. This resulted in a training quadrant time that was poor, yet significantly better than that of the HIPP-group, which displayed mainly "platform biased circling" throughout the transfer test and performed at chance (see above). The rapid behavioural shift by the RE-group indicates an interference with the suppression of strategy shifting, which normally occurs whenever the most effective strategy has been selected, and resembles old observations by Flämig and Klingberg (1978). They found no effects of a RE lesion on learning of a conditioned avoidance task in a Ymaze, but noticed a significant increase in anticipatory responses. In contrast to our initial hypothesis, we found no major hippocampal related effects of a RE lesion on standard reference memory watermaze learning. There is the possibility that $\mathrm{RE}$ is more involved in working memory (e.g., see Vann et al. 2000), and thus other tests would be more suited to investigate the effects of a RE lesion.

The initial phase of the transfer test of MD-rats was characterized by mainly loops to the platform location. This was followed by persistent searching in the training quadrant, which indicates that MD-rats had learned and remembered the correct location. Therefore, it is unlikely that the transient acquisition deficit of MD-rats was due to a deficit in learning of the task or in using spatial cues, but was initially due to perseveration of an ineffective strategy (i.e., thigmotaxis) and an impaired ability to switch to other strategies. In this respect, it might also be of interest to examine the effects of RE- and MD-lesions, using the egocentric response-learning version of the watermaze task (e.g., De Bruin et al. 2001).

The differences in the ability/readiness of RE- and MDlesioned rats to use strategies in a flexible way (i.e., RE-rats being very flexible; MD-rats showing perseveration), is further supported by our observations in the cue test. Specifically, there were no indications that any of the lesion groups had sensorimotor deficiencies, or a lack of motivation. HIPP-rats showed normal latencies compared to controls, as in previous studies (Morris et al. 1982; 
Pouzet et al. 2002), whereas RE-rats initially escaped onto the platform even faster than SH- and HIPP-rats. Although the MD-group had long latencies in the first trials, likely because of searching for the submerged platform, it improved across trials to the control level. In the cue test, the only intramaze cue is the platform itself, and therefore this task is far less demanding than the hidden platform version. Nevertheless, it still requires a shift in strategy, prompts the suppression of the previously learned response (i.e., swim to the hidden platform), and the subsequent selection and facilitation of a new response (i.e., swim to the visible platform). Our results show that the RE-lesioned rats were very fast in shifting to a new strategy, whereas the MD-group was probably hampered by initial perseveration of the previously learned hidden platform response. This suggests that, in contrast to RE-lesioned rats, MDlesioned rats have a problem in shifting strategy when task demands are altered (Kolb et al. 1982; Hunt and Aggleton 1998; Block et al. 2007).

An important qualification is that the observed effects of a HIPP-, RE- and MD-lesion appear to be primarily due to destruction of the intended area, with little if any contribution of (minor) damage in adjacent structures. In comparison, our RE lesions were more selective than the RE lesions in the study by Flämig and Klingberg (1978). Our MD lesions also appeared more selective than the ones described in previous reports (e.g., Kolb et al. 1982; Beracochea et al. 1989; Hunt and Aggleton, 1991; Cain et al. 2006), although a straightforward comparison is hampered by the use of different lesion methods (e.g., chemical, electrolytic, radiofrequency). We failed to observe any significant correlations between lesion size and two measures of performance-acquisition (as indicated by the use of relatively direct $\mathrm{E}+\mathrm{F}+\mathrm{G}$ paths, associated with short escape latencies), and memory for the platform location (as indicated by training quadrant time). It should, however, be mentioned that the variation in extent of the MD lesions showed a slight tendency towards a correlation on the acquisition measure (i.e., strategy use). This might suggest that rats with small MD lesions (in this study involving approximately $50 \%$ of MD) were more likely to use relatively direct strategies (i.e., suggestive for somewhat less perseverative behaviour) than rats with larger, near complete MD lesions.

Behavioural considerations in relation to thalamic-mPFC connectivity

Afferents from RE were shown to exert excitatory effects on mPFC, similar to actions in the hippocampus, and thus RE appears to be in a position to influence and/or coordinate activity in both systems (Dolleman-Van der Weel et al. 1997; Di Prisco and Vertes 2006). RE neurons, receiving input from $\mathrm{mPFC}$, have been shown to innervate the hippocampal CA1 area. Hence, RE is assumed to represent a critical link in a HIPP-mPFC-RE-HIPP neuronal circuitry (Vertes et al. 2007). Based on its strong reciprocal connections with the $\mathrm{mPFC}$, and the involvement of the latter area in behavioural flexibility, it has been suggested that RE might play a role in the selection of appropriate responses (Vertes 2006; Vertes et al. 2006). The present observations, however, show that rats in which RE is destroyed can rapidly display the most appropriate response in both the hidden platform and cue tasks. Therefore, it is unlikely that RE plays a critical role in response selection - at least not in these two tasks. Instead, our results yield a clue towards involvement of RE in shifting strategy. Kolb et al. (1982, 1983) showed that mPFC-lesioned rats can fail to learn a watermaze task. Later studies revealed that $\mathrm{mPFC}$ lesions can also cause no impairment in spatial navigation, but rather result in a deficit of behavioural flexibility (De Bruin et al. 1994, 2001; Lacroix et al. 2002). In various tests, it has been shown that destruction or inactivation of mPFC does not affect learning and memory per se, but impairs the animals' ability to shift strategy, or rule out inappropriate strategies when task demands are changed or environmental conditions are altered (Ragozzino et al. 1999a, b; Delatour and Gisquet-Verrier 2000; Dias and Aggleton 2000; Lacroix et al. 2002; Sullivan and Gratton 2002; Passetti et al. 2002; Ragozzino et al. 2003; Ragozzino 2007). At first sight, in relation to $\mathrm{RE}-\mathrm{mPFC}$ connectivity, these reports seem contradictory to the rapid strategy shifting by RE-rats. However, abnormalities in behavioural flexibility can be due to a disturbance of $\mathrm{mPFC}$-mediated inhibitory response control (e.g., Carli et al. 2006), resulting in (1) a failure of response inhibition, expressed as inappropriate anticipatory or "impulsive" responding (see RE-lesioned rats), or (2) a failure to suppress/inhibit an aimless repetition of an irrelevant response/strategy, causing perseverative behaviour (see MD-lesioned rats). Therefore, the ability of RElesioned rats to shift very rapidly from one strategy to another might have been due to the loss of excitatory RE input to $\mathrm{mPFC}$, causing a dysfunction of inhibitory response control mechanisms (e.g., Murphy et al. 2005). Hence, RE may be of importance for the suppression of (inappropriate/impulsive) strategy shifting, thereby opposing the role of MD (see below).

Only a few studies have used the watermaze to examine the effects of a MD or medial thalamus lesion on spatial learning (e.g., Kolb et al. 1982; Cain et al. 2006). A comparison with previous observations, however, is complicated due, for instance, to considerable differences in actual extent of the lesions, as well as in training and test procedures. In general, the deficits in watermaze performance resulting from a MD lesion resemble those seen 
after an mPFC lesion, i.e., little or no effect on spatial aspects, but slower acquisition and/or reversal training which is proposed to reflect deficits in non-mnemonic processes as reduced behavioural flexibility (e.g., Lacroix et al. 2002). Perseveration by MD-rats, or an impaired ability to shift strategies, appears to be a consistent finding and has been reported in various studies with a variety of tests (e.g., Stokes and Best 1988; Beracochea et al. 1989; McAlonan et al. 1993; Hunt and Aggleton 1991, 1998). Interestingly, Floresco and Grace (2003) showed that MDto-mPFC projection neurons exert a complex excitatoryinhibitory gating action over hippocampal input in PFC. They suggested that MD-PFC input may be able to facilitate or inhibit hippocampal input upon mPFC, supposedly permitting strategy switching by facilitation of a new strategy while at the same time inhibiting a previously learned one. More recently, using a cross-maze-based strategy set-shifting task, Block et al. (2007) provided further evidence that MD is involved in behavioural flexibility. Inactivation of $\mathrm{MD}$ disturbed the flow of information from MD-to-mPFC, resulting in a perseverative deficit. They proposed that the MD-to-mPFC connection may play a role in signaling the need to shift strategy. In turn, the mPFC then serves to suppress perseveration of the now irrelevant response. Our results appear in accordance with such a role for MD in strategy shifting.

In summary, while HIPP-lesions cause the expected deficit in the protocol used here, lesions of RE or MD did not prevent the learning or later memory of a standard watermaze task. Instead, lesions of RE or MD appeared to affect the normal flexible use of search strategies and/or the flexibility with which a change in task conditions can be accommodated (i.e., a RE lesion resulted in very flexible/ impulsive behaviour; a MD lesion caused perseverative behaviour). Based on the present observations, and in line with described modulatory effects of RE-to-PFC and MDto-PFC projections (Floresco and Grace 2003; Di Prisco and Vertes 2006), we hypothesize that RE and MD play opposing roles in non-mnemonic processes like strategy shifting, or in general aspects of behavioural flexibility. This hypothesis should be tested in future research, using electrophysiological methods and additional appropriate behavioural tests.

Acknowledgments We thank J. P. C. de Bruin and F. H. Lopes da Silva for stimulating discussions and reading of an early version of the manuscript. We gratefully acknowledge the technical assistance by the technicians at the Department of Neuroscience, University of Edinburgh, at the Department of Anatomy, VU University Medical Centre, Amsterdam, and at the Netherlands Institute for Neuroscience, Amsterdam. We also thank G. Docter for helping with statistical analyses, and D. de Jong for processing of the microphotographs. This work was supported by NWA Grant 90-20 from the Graduate School for Neurosciences Amsterdam.
Open Access This article is distributed under the terms of the Creative Commons Attribution Noncommercial License which permits any noncommercial use, distribution, and reproduction in any medium, provided the original author(s) and source are credited.

\section{References}

Aggleton JP, Vann SD, Oswald CJ, Good M (2000) Identifying cortical inputs to the rat hippocampus that subserve allocentric spatial processes: a simple problem with a complex answer. Hippocampus 10:466-474. doi:10.1002/1098-1063(2000)10:4 $<466$ ::AID-HIPO13>3.0.CO;2-Y

Baxter MG, Murphy KL, Crosby G, Culley DJ (2008) Different behavioural effects of neurotoxic dorsal hippocampal lesions placed under either isoflurane or propofol anesthesia. Hippocampus 18:245-250. doi:10.1002/hipo.20390

Beracochea DJ, Jaffard R, Jarrard LE (1989) Effects of anterior or dorsomedial thalamic ibotenic lesions on learning and memory in rats. Behav Neural Biol 51:364-376. doi:10.1016/S0163-1047 (89)91000-5

Bertaina-Anglade V, Tramu G, Destrade C (2000) Differential learning-stage dependent patterns of c-Fos protein expression in brain regions during the acquisition and memory consolidation of an operant task in mice. Eur J Neurosci 12:3803-3812. doi:10.1046/j.1460-9568.2000.00258.x

Block AE, Dhanji H, Thompson-Tardif SF, Floresco SB (2007) Thalamic-prefrontal cortical-ventral striatal circuitry mediates dissociable components of strategy set shifting. Cereb Cortex 17:1625-1636. doi:10.1093/cercor/bh1073

Braak H, Braak E (1991) Alzheimer's disease affects limbic nuclei of the thalamus. Acta Neuropathol 81:261-268. doi:10.1007/ BF00305867

Cain PP, Boon F, Corcoran M (2006) Thalamic and hippocampal mechanisms in spatial navigation: a dissociation between brain mechanisms for learning how versus learning where to navigate. Behav Brain Res 170:241-256. doi:10.1016/j.bbr.2006.02.023

Carli M, Baviera M, Invernizzi RW, Balducci C (2006) Dissociable contribution of $5-\mathrm{HT}_{1 \mathrm{~A}}$ and $5-\mathrm{HT}_{2 \mathrm{~A}}$ receptors in the medial prefrontal cortex to different aspects of executive control such as impulsivity and compulsive perseveration in rats. Neuropsychopharmacol 31:757-767. doi:10.1038/sj.npp.1300893

Chauveau F, Celerier A, Ognard R, Pierard C, Beracochea D (2005) Effects of ibotenic acid lesions of the mediodorsal thalamus on memory: relationship with emotional processes in mice. Behav Brain Res 156:215-223. doi:10.1016/j.bbr.2004.05.026

Culley DJ, Baxter M, Yukhananov R, Crosby G (2003) The memory effects of general anesthesia persist for weeks in young and aged rats. Anesth Analg 96:1004-1009. doi:10.1213/01.ANE. 0000052712.67573 .12

Culley DJ, Baxter MG, Yukhananov R, Crosby G (2004) Long-term impairment of acquisition of a spatial memory task following isoflurane-nitrous oxide anesthesia in rats. Anesthesiology 100:309-314. doi:10.1097/00000542-200402000-00020

De Bruin JPC, Sanchez-Santed F, Heinsbroek RPW, Donker A, Postmes P (1994) A behavioural analysis of rats with damage to the medial prefrontal cortex using the Morris water maze: evidence for behavioural flexibility, but not for impaired spatial navigation. Brain Res 652:323-333. doi:10.1016/0006-8993(94) 90243-7

De Bruin JP, Moita MP, de Brabander HM, Joosten RN (2001) Place and response learning of rats in a Morris water maze: differential effects of fimbria fornix and medial prefrontal cortex lesions. Neurobiol Learn Mem 75:164-178. doi:10.1006/nlme.2000.3962 
Delatour B, Gisquet-Verrier P (2000) Functional role of rat prelimbic-infralimbic cortices in spatial memory: evidence for their involvement in attention and behavioural flexibility. Behav Brain Res 109:113-128. doi:10.1016/S0166-4328(99) 00168-0

Dias R, Aggleton JP (2000) Effects of selective excitotoxic prefrontal lesions on acquisition of nonmatching- and matching-to-place in the T-maze in the rat: differential involvement of the prelimbicinfralimbic and anterior cingulate cortices in providing behavioural flexibility. Eur J Neurosci 12:4457-4466. doi:10.1046/j. 0953-816X.2000.01323.x

Di Prisco GV, Vertes RP (2006) Excitatory actions of the ventral midline thalamus (rhomboid/reuniens) on the medial prefrontal cortex in the rat. Synapse 60:45-55. doi:10.1002/syn.20271

Dolleman-van der Weel MJ, Witter MP (1996) Projections from the nucleus reuniens thalami to the entorhinal cortex, hippocampal field CA1, and the subiculum in the rat arise from different populations of neurons. J Comp Neurol 364:637-650. doi:10. 1002/(SICI)1096-9861(19960122)364:4<637::AID-CNE3>3.0. $\mathrm{CO} ; 2-4$

Dolleman-van der Weel MJ, Lopes da Silva FH, Witter MP (1997) Nucleus reuniens thalami modulates activity in hippocampal field CA1 through excitatory and inhibitory mechanisms. J Neurosci 17:5640-5650

Dolleman-van der Weel MJ, Witter MP (2000) Nucleus reuniens thalami innervates gamma aminobutyric acid positive cells in hippocampal field CA1 of the rat. Neurosci Lett 278:145-148. doi:10.1016/S0304-3940(99)00935-0

Flämig R, Klingberg F (1978) Die Beteiligung thalamischer Kerne an der Ausbildung bedingter Fluchtreflexe der Ratte IV. Läsionen des nucleus reuniens. Acta Biol Med Ger 37:1779-1782

Floresco SB, Grace AA (2003) Gating of hippocampal-evoked activity in prefrontal cortical neurons by inputs from the mediodorsal thalamus and ventral tegmental area. J Neurosci 23:3930-3943

Gerlai RT, NcNamara A, Williams S, Philips HS (2002) Hippocampal dysfunction and behavioural deficit in the water maze in mice: An unresolved issue? Brain Res Bull 57:3-9. doi:10.1016/ S0361-9230(01)00630-X

Groenewegen HJ (1988) Organization of the afferent connections of the mediodorsal nucleus in the rat, related to the mediodorsalprefrontal topography. Neurosci 24:379-431. doi:10.1016/03064522(88)90339-9

Herkenham M (1978) The connections of the nucleus reuniens thalami: evidence for a direct thalamohippocampal pathway. J Comp Neurol 177:589-609. doi:10.1002/cne.901770405

Herrera DG, Robertson HA (1996) Activation of c-fos in the brain. Prog Neurobiol 50:83-107. doi:10.1016/S0301-0082(96) 00021-4

Hunt PR, Aggleton JP (1991) Medial dorsal thalamic lesions and working memory in the rat. Behav Neural Biol 55:227-246. doi: 10.1016/0163-1047(91)80141-Z

Hunt PR, Aggleton JP (1998) Neurotoxic lesions of the dorsomedial thalamus impair acquisition but not the performance of delayed matching to place by rats: a deficit in shifting response rules. J Neurosci 18:10045-10052

Jarrard LE (1989) On the use of ibotenic acid to lesion selectively different components of the hippocampal formation. J Neurosci Methods 29:251-259. doi:10.1016/0165-0270(89)90149-0

Jenkins TA, Amin E, Harold GT, Pearce JM, Aggleton JP (2003) Distinct patterns of hippocampal formation activity associated with different spatial tasks: a Fos imaging study in rats. Exp Brain Res 151:514-523. doi:10.1007/s00221-003-1499-0

Köhler C, Schwarcz R (1983) Comparison of ibotenate and kainate neurotoxicity in rat brain: a histological study. Neuroscience 8:819-835. doi:10.1016/0306-4522(83)90013-1
Kolb B, Pittman K, Sutherland RJ, Whishaw IQ (1982) Dissociation of the contributions of the prefrontal cortex and dorsomedial thalamic nucleus to spatially guided behavior in the rat. Behav Brain Res 6:365-378. doi:10.1016/0166-4328(82)90018-3

Kolb B, Sutherland RJ, Whishaw IQ (1983) A comparison of the contributions of the medial frontal and parietal association cortex to spatial localization in rats. Behav Neurosci 97:13-27. doi: 10.1037/0735-7044.97.1.13

Krettek JE, Price JL (1977) The cortical projections of the mediodorsal nucleus and adjacent thalamic nuclei in the rat. J Comp Neurol 171:157-192. doi:10.1002/cne.901710204

Lacroix I, White I, Feldon J (2002) Effect of excitotoxic lesions of rat medial prefrontal cortex on spatial memory. Behav Brain Res 133:69-81. doi:10.1016/S0166-4328(01)00442-9

Lieggi CC, Artwohl JE, Leszcynski JK, Rodriguez NA, Fickbohm BL, Fortmann JD (2005) Efficacy and safety of stored and newly prepared tribromoethanol in ICR mice. Contemp Top Lab Anim Sci 44:17-22

Markowitsch HJ (1982) Thalamic mediodorsal nucleus and memory: a critical evaluation of studies in animal and man. Neurosci Biobehav Rev 6:351-380. doi:10.1016/0149-7634(82)90046-X

McAlonan GM, Robbins HE, Everitt BJ (1993) Effects of medial dorsal thalamic and ventral pallidal lesions on the acquisition of a conditioned place preference: further evidence for the involvement of the ventral striatopallidal system in rewardrelated processes. Neuroscience 52:605-620. doi:10.1016/03064522(93)90410-H

McKenna JT, Vertes RP (2004) Afferent projections to nucleus reuniens of the thalamus. J Comp Neurol 480:115-142. doi: $10.1002 / \mathrm{cne} .20342$

Meyer RE, Fisch RE (2005) A review of tribromoethanol anesthesia for production of genetically engineered mice and rats. Lab Anim (NY) 34:47-52. doi:10.1038/laban1105-47

Mitchell AS, Browning PG, Baxter MG (2007a) Neurotoxic lesions of the mediodorsal nucleus of the thalamus disrupt reinforcer devaluation effects in rhesus monkeys. J Neurosci 27:1128911295. doi:10.1523/JNEUROSCI.1914-07.2007

Mitchell AS, Baxter MG, Gaffan D (2007b) Dissociable performance on scene learning and strategy implementation after lesions to magnocellular mediodorsal thalamic nucleus. J Neurosci 31:11888-11895. doi:10.1523/JNEUROSCI.1835-07.2007

Morris RGM, Garrud P, Rawlins JNP (1982) Place navigation impaired in rats with hippocampal lesions. Nature 297:681-683. doi:10.1038/297681a0

Morris RGM, Schenk F, Tweedie F, Jarrard LE (1990) Ibotenate lesions of hippocampus and/or subiculum: dissociating components of allocentric spatial learning. Eur J Neurosci 2:10161028. doi:10.1111/j.1460-9568.1990.tb00014.x

Moser E, Moser M-B, Andersen P (1993) Spatial learning impairment parallels the magnitude of dorsal hippocampal lesions, but is hardly present following ventral lesions. J Neurosci 13:3916-3925

Moser M-B, Moser EI (1998) Distributed encoding and retrieval of spatial memory in the hippocampus. J Neurosci 15:7535-7542

Murphy ER, Daley JW, Robbins TW (2005) Local glutamate receptor antagonism in the rat prefrontal cortex disrupts response inhibition in a visuospatial attentional task. Psychopharmacology 179:99-107. doi:10.1007/s00213-004-2068-3

Passetti F, Chudasama Y, Robbins TW (2002) The frontal cortex of the rat and visual attentional performance: dissociable functions of distinct medial prefrontal subregions. Cereb Cortex 12:12541268. doi:10.1093/cercor/12.12.1254

Paxinos G, Watson C (1986) The rat brain in stereotaxic coordinates. Academic Press, Sidney

Peinado-Manzano MA, Pozo-Garcia R (1996) Retrograde amnesia in rats with dorsomedial thalamic damage. Behav Brain Res 80:177-184. doi:10.1016/0166-4328(96)00033-2 
Pouzet B, Zhang WN, Feldon J, Rawlins JN (2002) Hippocampal lesioned rats are able to learn a spatial position using non-spatial strategies. Behav Brain Res 133:279-291. doi:10.1016/S01664328(02)00007-4

Ragozzino ME, Detrick S, Kesner RP (1999a) Involvement of the prelimbic-infralimbic areas of the rodent prefrontal cortex in behavioral flexibility for place and response learning. J Neurosci 19:4585-4594

Ragozzino ME, Wilcox C, Raso M, Kesner RP (1999b) Involvement of rodent prefrontal cortex subregions in strategy switching. Behav Neurosci 113:32-41. doi:10.1037/0735-7044.113.1.32

Ragozzino ME, Kim J, Hasser D, Minniti N, Kiang C (2003) The contribution of the rat prelimbic-infralimbic areas to different forms of task switching. Behav Neurosci 117:1054-1065. doi: 10.1037/0735-7044.117.5.1054

Ragozzino ME (2007) The contribution of the medial prefrontal cortex, orbitofrontal cortex, and dorsomedial striatum to behavioral flexibility. Ann N Y Acad Sci 1121:355-375. doi: 10.1196/annals. 1401.013

Rotaru DC, Barrionuevo G, Sesack SR (2005) Mediodorsal thalamic afferents to layer III of the rat prefrontal cortex: synaptic relationships to subclasses of interneurons. J Comp Neurol 490:220-238. doi:10.1002/cne.20661

Rousseau M (1994) Amnesia following limited thalamic lesions. In Delacour J (ed) The memory system of the brain, vol 4. World Scientific Publishers, Singapore.Adv. Series Neurosci, pp 241277

Santin LJ, Aguirre JA, Rubio S, Miranda R, Arias JL (2003) c-Fos expression in supramammillary and medial mammillary nuclei following spatial reference and working memory tasks. Physiol Behav 78:733-739. doi:10.1016/S0031-9384(03)00060-X

Stokes KA, Best PJ (1988) Mediodorsal thalamic lesions impair radial maze performance in the rat. Behav Neurosci 102:294-300. doi: 10.1037/0735-7044.102.2.294

Stokes KA, Best PJ (1990) Mediodorsal thalamic lesions impair reference and working memory in rats. Physiol Behav 47:471476. doi:10.1016/0031-9384(90)90111-G

Sullivan RM, Gratton A (2002) Behavioral effects of excitotoxic lesions of ventral medial prefrontal cortex in the rat are hemisphere-dependent. Brain Res 927:69-79. doi:10.1016/ S0006-8993(01)03328-5

Thompson JS, Brown SA, Khurdayan V, Zeynalzadedan A, Sullivan PG, Scheff SW (2002) Early effects of tribromoethanol, ketamine/xylazine, pentobarbital, and isoflurane anesthesia on hepatic and lymphoid tissue in ICR mice. Comp Med 52:63-67

Van der Werf YD, Witter MP, Uylings HBM, Jolles J (2000) Neuropsychology of infarctions in the thalamus: a review. Neuropsychologia 38:613-627. doi:10.1016/S0028-3932(99) 00104-9
Van der Werf YD, Jolles J, Witter MP, Uylings HB (2003) Contributions of thalamic nuclei to declarative memory functioning. Cortex 39:1047-1062. doi:10.1016/S0010-9452(08) 70877-3

Vann SD, Brown MW, Aggleton JP (2000) Fos expression in the rostral thalamic nuclei and associated cortical regions in response to different spatial memory tests. Neuroscience 101:983-991. doi:10.1016/S0306-4522(00)00288-8

Vertes RP (2002) Analysis of projections from the medial prefrontal cortex to the thalamus in the rat, with emphasis on nucleus reuniens. J Comp Neurol 442:163-187. doi:10.1002/cne.10083

Vertes RP (2004) Differential projections of the infralimbic and prelimbic cortex in the rat. Synapse 51:32-58. doi:10.1002/syn. 10279

Vertes RP (2006) Interactions among the medial prefrontal cortex, hippocampus and midline thalamus in emotional and cognitive processing in the rat. Neuroscience 142:1-20. doi:10.1016/j. neuroscience.2006.06.027

Vertes RP, Hoover WB, do Valle AC, Sherman A, Rodriquez JJ (2006) Efferent projections of reuniens and rhomboid nuclei of the thalamus in the rat. J Comp Neurol 499:768-796. doi: $10.1002 /$ cne. 21135

Vertes RP, Hoover WB, Szigeti-Buck K, Leranth C (2007) Nucleus reuniens of the midline thalamus: link between the medial prefrontal cortex and the hippocampus. Brain Res Bull 71:601609. doi:10.1016/j.brainresbull.2006.12.002

Whishaw IO, Jarrard LE (1995) Similarities vs. differences in place learning and circadian activity in rats after fimbria-fornix section or ibotenate removal of hippocampal cells. Hippocampus 5:595604. doi:10.1002/hipo.450050610

Whishaw IQ, Cassel J-C, Jarrard LE (1995) Rats with fimbria-fornix lesions display a place response in a swimming pool: a dissociation between getting there and knowing where. J Neurosci 15:5779-5788

Whishaw IQ, Tomie J-A (1997) Perseveration on place reversals in spatial swimming pool tasks: further evidence for place learning in hippocampal rats. Hippocampus 7:361-370. doi:10.1002/ (SICI) 1098-1063(1997)7:4<361::AID-HIPO2>3.0.CO;2-M

Wouterlood FG, Saldana E, Witter MP (1990) Projections from the nucleus reuniens thalami to the hippocampal region: light and electron microscopic tracing study in the rat with the anterograde tracer Phaseolus vulgaris-Leucoagglutinin. J Comp Neurol 296:179-203. doi:10.1002/cne.902960202

Zeller W, Meier G, Burki K, Panoussis B (1998) Adverse effects of tribromoethanol as used in the production of transgenic mice. Lab Anim 32:407-413. doi:10.1258/002367798780599811

Zhang J, McQuade JM, Vorhees CV, Xu M (2002) Hippocampal expression of c-fos is not essential for spatial learning. Synapse 46:91-99. doi:10.1002/syn.10115 